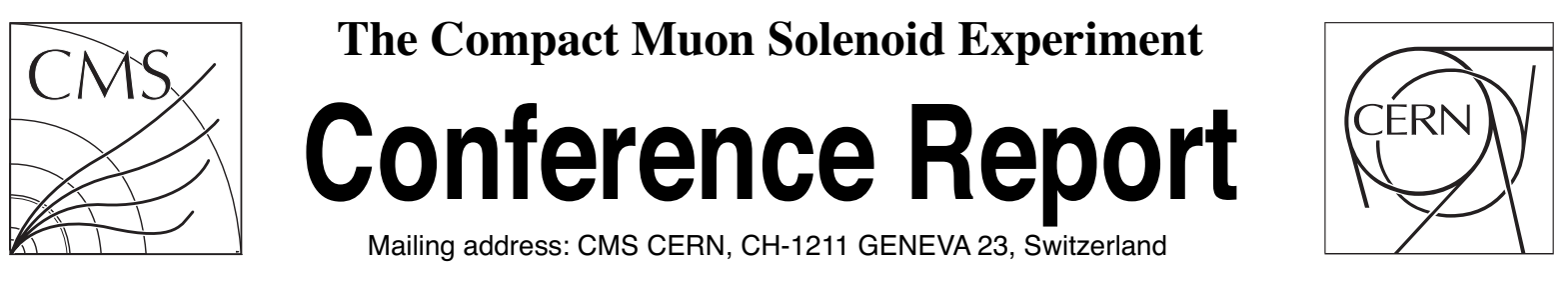

07 December 2017 (v4, 14 January 2018)

\title{
Performance of the CMS precision electromagnetic calorimeter at LHC Run II and prospects for High-Luminosity LHC
}

Zhicai Zhang for the CMS Collaboration

\begin{abstract}
Many physics analyses using the Compact Muon Solenoid (CMS) detector at the LHC require accurate, high-resolution electron and photon energy measurements. Following the excellent performance achieved during LHC Run I at center-of-mass energies of 7 and $8 \mathrm{TeV}$, the CMS electromagnetic calorimeter (ECAL) is operating at the LHC with proton-proton collisions at $13 \mathrm{TeV}$ center-of-mass energy. The instantaneous luminosity delivered by the LHC during Run II has achieved unprecedented levels. The average number of concurrent proton-proton collisions per bunch-crossing (pileup) has reached up to 40 interactions in 2016 and may increase further in 2017. These high pileup levels necessitate a retuning of the ECAL readout and trigger thresholds and reconstruction algorithms. In addition, the energy response of the detector must be precisely calibrated and monitored. We present new reconstruction algorithms and calibration strategies that were implemented to maintain the excellent performance of the CMS ECAL throughout Run II. We will show performance results from the 2015-2016 data taking periods and provide an outlook on the expected Run II performance in the years to come. Beyond the LHC, challenging running conditions for CMS are expected after the High-Luminosity upgrade of the LHC (HL-LHC). We review the design and R and D studies for the CMS ECAL and present first test beam studies. Particular challenges at HL-LHC are the harsh radiation environment, the increasing data rates, and the extreme level of pile-up events, with up to 200 simultaneous proton-proton collisions. We present test beam results of hadron irradiated PbWO crystals up to fluences expected at the HL-LHC. We also report on the R and D for the new readout and trigger electronics, which must be upgraded due to the increased trigger and latency requirements at the HL-LHC.
\end{abstract}




\title{
Performance of the CMS precision electromagnetic calorimeter at LHC Run II and prospects for High-Luminosity LHC
}

\author{
Zhicai Zhang ${ }^{a}$ on behalf of the CMS collaboration \\ ${ }^{a}$ California Institute of Technology, \\ 1200 E. California Blvd, Pasadena, CA 91125, USA \\ E-mail: zzhang2@caltech.edu
}

Abstract: Many physics analyses using the Compact Muon Solenoid (CMS) detector at the LHC require accurate, high-resolution electron and photon energy measurements. Following the excellent performance achieved during LHC Run I at center-of-mass energies of 7 and $8 \mathrm{TeV}$, the CMS electromagnetic calorimeter (ECAL) is operating at the LHC with proton-proton collisions at $13 \mathrm{TeV}$ center-of-mass energy. The instantaneous luminosity delivered by the LHC during Run II has achieved unprecedented levels. The average number of concurrent proton-proton collisions per bunch-crossing (pileup) has reached up to 40 interactions in 2016 and may increase further in 2017. These high pileup levels necessitate a retuning of the ECAL readout and trigger thresholds and reconstruction algorithms. In addition, the energy response of the detector must be precisely calibrated and monitored. We present new reconstruction algorithms and calibration strategies that were implemented to maintain the excellent performance of the CMS ECAL throughout Run II. We will show performance results from the 2015-2016 data taking periods and provide an outlook on the expected Run II performance in the years to come.

Beyond the LHC, challenging running conditions for CMS are expected after the HighLuminosity upgrade of the LHC (HL-LHC). We review the design and R\&D studies for the CMS ECAL and present first test beam studies. Particular challenges at HL-LHC are the harsh radiation environment, the increasing data rates, and the extreme level of pile-up events, with up to 200 simultaneous proton-proton collisions. We present test beam results of hadron irradiated PbWO crystals up to fluences expected at the HL-LHC. We also report on the R\&D for the new readout and trigger electronics, which must be upgraded due to the increased trigger and latency requirements at the HL-LHC.

Keywords: Performance of High Energy Physics Detectors; Calorimeters; Calibration methods 


\section{Contents}

1 Introduction 1

2 The CMS ECAL 1

3 Reconstruction, calibration and performance of ECAL at the LHC Run II 1

4 Prospects for high-luminosity LHC 4

5 Summary 5

\section{Introduction}

The Compact Muon Solenoid (CMS) experiment has produced a broad range of physics results by exploiting the proton-proton and heavy-ion collision data at the Large Hadron Collider (LHC), including the discovery of the standard model (SM) Higgs boson, as well as searches for physics beyond SM. The CMS electromagnetic calorimeter (ECAL) is one of the crucial sub-detectors for the success of CMS, and measures the energy of $e / \gamma$ particles with excellent resolution and also provides position measurements for them. In this report, we will present the performance of the ECAL with $\sqrt{s}=13 \mathrm{TeV}$ collision data along with the reconstruction algorithms and calibration strategies used to obtain such performance. We will also discuss the upgrade strategies of ECAL that have been proposed to maintain the performance at high-luminosity LHC.

\section{The CMS ECAL}

The CMS ECAL is a homogeneous and hermetic electromagnetic calorimeter made of 75848 lead tungstate $\left(\mathrm{PbWO}_{4}\right)$ scintillating crystals, covering a pseudorapidity region up to $\eta=1.479$ (3.0) in the barrel (endcap). The scintillation light from the crystal is detected by Avalanche Photo-Diodes (APD) in the barrel and Vacuum Phototriodes (VPT) in the endcap. Further technical design details of the CMS ECAL can be found in reference [1]. For high energy $e / \gamma$ from $\mathrm{Z}$ or Higgs boson decays, the CMS ECAL achieved an energy resolution as good as $1 \%$ for photons in the central barrel region. This excellent energy resolution was crucial for the discovery of the Higgs boson in the final states with two photons or four leptons.

\section{Reconstruction, calibration and performance of ECAL at the LHC Run II}

Since the transverse size of a $\mathrm{PbWO}_{4}$ crystal in ECAL is approximately equal to its MoliÃĺre radius, deposits from high energy electromagnetic showers are typically spread over several crystals and clustered with dedicated algorithms. The algorithm starts with a basic cluster, which is the local 
maximum of the energy deposited. Due to the material in front of ECAL and the strong magnetic field, photons convert and electrons emit bremsstrahlung photons, further resulting in a spread of the energy in the transverse plane. Such energy spread is recovered by extending the basic clusters to a supercluster. The total energy of an electron or photon is given by the sum over all crystals in the supercluster:

$$
E_{e, \gamma}=F_{e, \gamma} \cdot G \cdot \sum_{i} S_{i}(t) \cdot C_{i} \cdot A_{i}
$$

This sum starts from the raw pulse amplitude $A_{i}$ and takes several steps of corrections/calibrations to get the final reconstructed energy, as explained below.

Each pulse from the APD/VPT is digitized into 10 time samples. During LHC Run I, the pulse amplitude was taken as a weighted sum of all 10 samples. At LHC Run II, however, with the bunch spacing changed from $50 \mathrm{~ns}$ to $25 \mathrm{~ns}$ and with much higher instantaneous luminosity, the out-of-time (OOT) pileup (number of concurrent interactions per LHC bunch crossing) contamination to the in-time pulse becomes more severe. To mitigate the effect of such OOT pulses, a multifit method is adopted. The pulse is modeled as an in-time pulse plus several OOT pulses (up to 9), and the in-time pulse is obtained by performing a template fit.

Due to radiation damage, the ECAL crystal response changes with time, and partially recovers through self-annealing during periods without radiation. The response change in ECAL, denoted by $S_{i}(t)$ in eq.3.1, is continuously measured by a laser monitoring system by injecting laser light and measures the response of each channel; the corrections are then delivered in less than 48 hours for the prompt reconstruction of the data. The measured relative response to laser light from the start of the LHC running until May 2017, along with the LHC instantaneous luminosity, is shown in Fig. 1. The laser monitoring system is then validated with collision data by looking at the time stability of the invariant mass of $\pi^{0}$ decays to two photons, and the ratio of electron (from $\mathrm{W}$ or $\mathrm{Z}$ boson) energy $E$ (measured by ECAL) to its momentum $p$ (measured by tracker). Fig. 2 shows the normalized $\pi^{0}$ mass before and after applying the response correction for $\pi^{0}$ in the ECAL barrel during one fill of LHC, in which the stability of the mass peak is at the level of $0.19 \%$ RMS after the correction.

The relative calibration, denoted by inter-calibration constant $C_{i}$, equalizes the different crystal+APD/VPT responses among different channels using the following three main methods:

$\phi$-symmetry: the energy flow in a constant $\eta$ ring is supposed to be uniform, thus the $C_{i}$ is obtained by equalizing the average transverse energy in crystals at constant $\eta$;

$\pi^{0}$ and $\eta$ mass: this method utilizes the invariant mass of $\pi^{0}(\eta) \rightarrow \gamma \gamma$ decays, which is reconstructed from the energy of the two photons; it is an iterative procedure in which the $C_{i}$ of the central crystal in the photon cluster gets updated in each iteration based on the reconstructed invariant mass and the iteration stops when the the precision converges;

$E / p$ : this is also an iterative method based on ECAL energy and tracker momentum for isolated electrons from $\mathrm{Z}$ or $\mathrm{W}$ boson decays.

The final set of $C_{i}$ is then taken as a weighted combination of the above three methods. The residual mis-calibration of the combined inter-calibration, is about $0.5 \%$ in the center of the detector and up to $1 \%$ in the high $\eta$ region of the barrel, as shown in the left plot of Fig. 3 


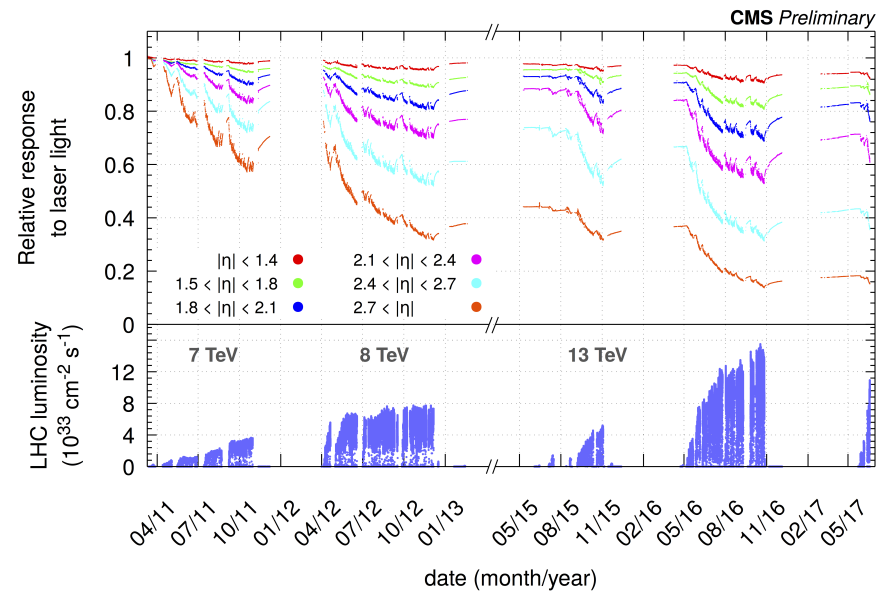

Figure 1. Relative response to laser light injected in the ECAL crystals, measured by the ECAL laser monitoring system, averaged over all crystals in bins of pseudorapidity, for the 2011, 2012, 2015, 2016 and 2017 data taking periods, with magnetic field at $3.8 \mathrm{~T}$.

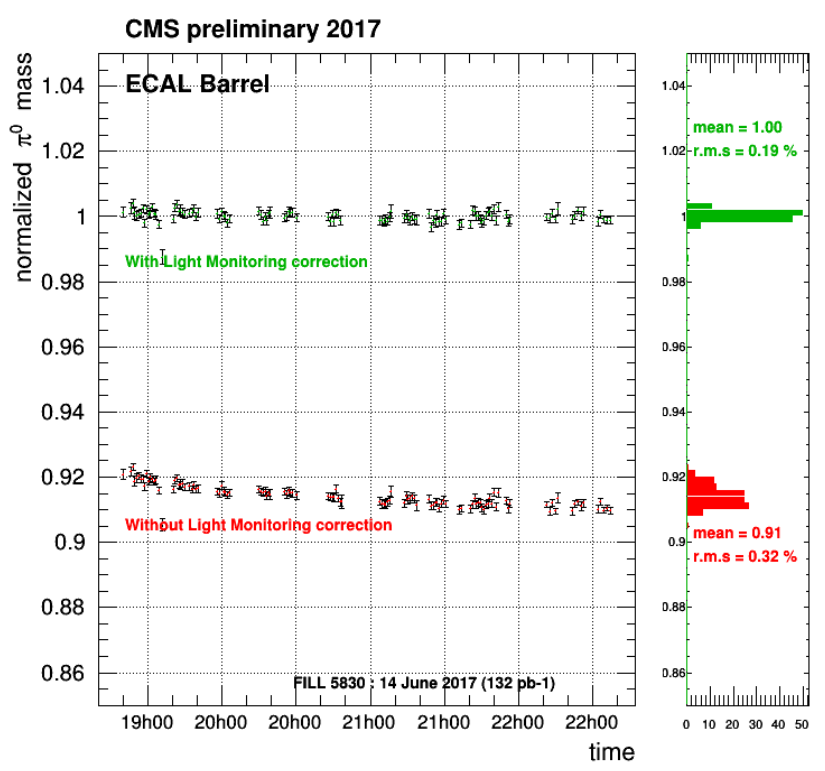

Figure 2. Stability of the relative energy scale measured from the peak of the invariant mass distribution of $\pi^{0} \rightarrow \gamma \gamma$ decays in the ECAL barrel. 

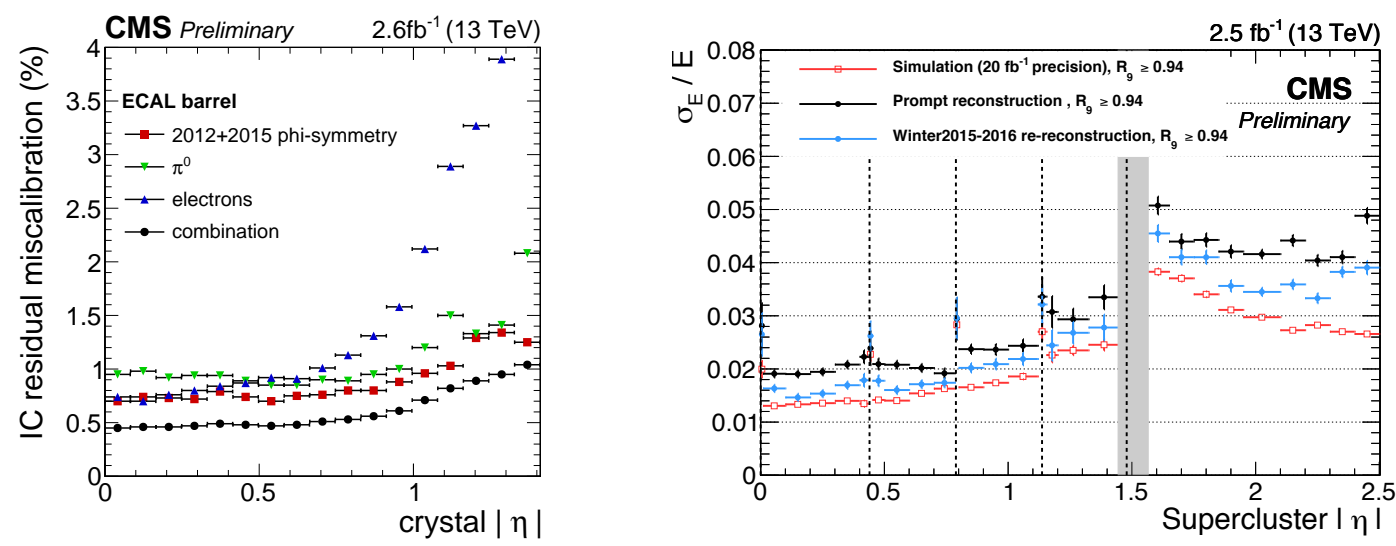

Figure 3. Left: Residual mis-calibration of the ECAL barrel (EB) channel inter-calibration, as a function of pseudo-rapidity with the dataset recorded during 2015. Right: Relative electron (ECAL) energy resolution distributed in bins of $\eta$ for the barrel and the endcaps. Low bremsstrahlung electrons $\left(R 9=E_{3 \times 3} / E_{S C}>0.94\right)$ from $\mathrm{Z}$ boson decays are used.

The raw energy of a supercluster calculated from the sum of individual hits, is further corrected for the material budget in front of the ECAL, as well as partial shower containment due to gaps and cracks between crystals. Such supercluster energy corrections, denoted by $F_{e, \gamma}$, are derived by a multivariate technique trained with simulated events in which the true energy is known, and is then validated with $Z \rightarrow e e$ events in collision data.

The last correction applied to the $e / \gamma$ energy is the absolute energy scale, $G$, which is obtained by matching the invariant mass distributions of $Z \rightarrow e e$ events in data to that of the simulation separately for barrel and endcap.

The single electron energy resolution of the ECAL is measured by relating it to the di-electron mass resolution of $Z \rightarrow e e$ events. The right plot of Fig. 3 shows the resolution in different $\eta$ bins, for both data and simulation, in which the simulated events assume the calibration precision that was achieved with the amount of data collected in Run 1. For the data, two sets of resolutions are shown in the plot - the black points correspond to the resolution with the calibration derived in 2012, which is significantly improved after combination with the calibration obtained with 2015 dataet, shown in the blue points. The best energy resolution achieved with $2.5 \mathrm{fb}^{-1}$ data taken in 2015 is between $1 \%$ and $2 \%$ in the central region of the detector, which is at the level of Run 1 , while in the endcaps the resolution is worse due to limited amount of data.

\section{Prospects for high-luminosity LHC}

With the high-luminosity upgrade of the current LHC, the CMS ECAL will be facing more challenging running conditions, with 5 times higher luminosity and much harsher radiation levels. The left plot of Fig. 4 shows the energy resolution of irradiated $\mathrm{PbWO}_{4}$ crystals measured in the test beam data with high energy electrons. The plot shows that for radiation levels in the barrel (corresponds to the blue line, triangles), the crystals will remain well performing, but an upgrade to the electronics and a decrease of the operating temperature will be required to maintain the current 
performance level. For the endcap crystals, the radiation damage is so severe (black line, crosses) as to necessitate a full replacement.
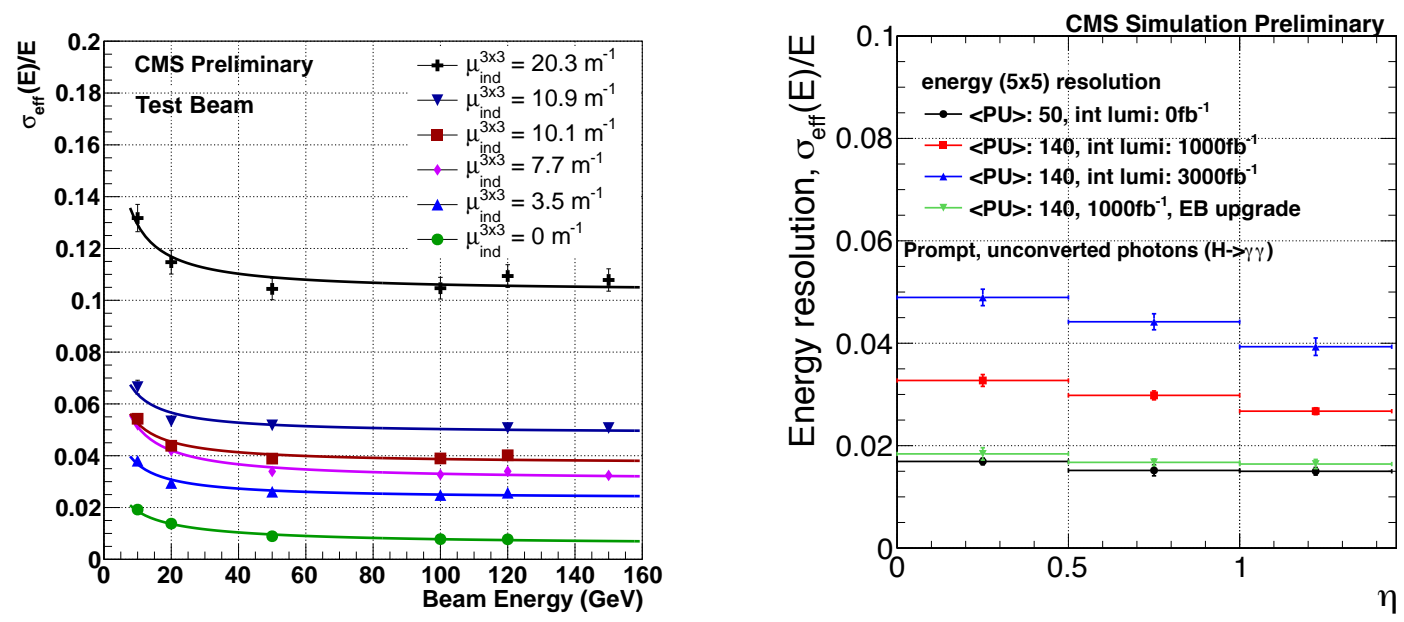

Figure 4. Left: Energy resolution of proton-irradiated $\mathrm{PbWO}_{4}$ crystals; the numbers in the legend indicate the average induced absorption, defined as $\mu_{\mathrm{IND}}(\lambda)=\frac{1}{\lambda} \times \ln \frac{L T_{0}(\lambda)}{L T(\lambda)}$, where $L T$ is the longitudinal light transmission at the wavelength $\lambda, L T_{0}$ is its value before irradiation, and $l$ is the crystal length. Right: Energy resolution based on simulation for $H \rightarrow \gamma \gamma$ photons at different pileup levels and for different integrated luminosities, with the upgrade of barrel ECAL.

For the HL-LHC upgrade of the ECAL barrel [2], the APDs along with the motherboards will remain unchanged. The APD dark current will increase at the HL-LHC due to increased radiation level; to mitigate the increase in noise, the upgraded ECAL will be operated in a colder environment (from current $18^{\circ} \mathrm{C}$ to $8^{\circ} \mathrm{C}$ ). The VFE will be replaced with optimized shaping time and increased sampling rate (from current $40 \mathrm{MHz}$ to $160 \mathrm{MHz}$ ) to reduce OOT pile-up, the impact of anomalous signals induced by direction ionization in APDs (spikes), dark current driven APD, and to improve the timing resolution. The FE will become a streaming readout and the data reduction at VFE and FE will be removed, moving most data processing off-detector. The respective links and offdetector electronics will therefore be upgraded to cope with such increased data volume and rate. The generation of the ECAL trigger primitives will also be performed off-detector, allowing the trigger decision to be made based on single channel information (instead of current $5 \times 5$ channel trigger towers) for improved spike rejection and trigger performance. The right plot of Fig. 4 shows the energy resolution of photons from Higgs decays before and after the upgrade of the ECAL barrel at the foreseen pileup level of HL-LHC. The energy resolution will be restored to the current performance at LHC Run II, which is also shown in the plot. The timing resolution of the CMS ECAL has been measured in test beams at CERN with the VFE prototype and increased sampling rate. The resolution at $160 \mathrm{MHz}$ digitization frequency for an effective amplitude of $A / \sigma=250$ (where $A$ is the amplitude and $\sigma$ is the width of the noise distribution) is measured to be $30 \mathrm{ps,}$ which corresponds to 25 (60) GeV photons at the start (end) of HL-LHC running, as shown in Fig. 5. No significant improvement of timing resolution is observed beyond $160 \mathrm{MHz}$, which sets 160 $\mathrm{MHz}$ as the current baseline of the upgrade. 


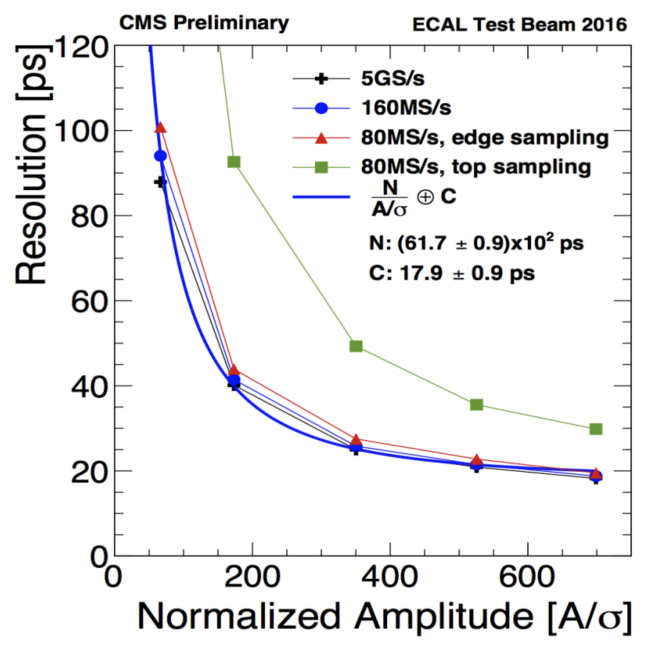

Figure 5. Timing resolution as a function of effective amplitude $A / \sigma$ where $\sigma$ is the width of the noise distribution, measured in test beam with a VFE prototype for upgraded barrel ECAL. The time resolution for different sampling rates are also shown, demonstrating that a sampling rate of $160 \mathrm{MHZ}$ will result in sufficient timing performance.

\section{Summary}

With new reconstruction and calibration methods adopted for LHC Run II, the CMS ECAL has achieved excellent performance, with single $e / \gamma$ energy resolution as low as $1 \%$ to $2 \%$ in the barrel. As the running conditions further change at the upcoming HL-LHC, the endcap of the ECAL must be totally replaced and a partial upgrade is planned for the barrel ECAL to maintain its performance.

\section{References}

[1] CMS Collaboration, The CMS electromagnetic calorimeter project: Technical Design Report, CERN LHCC-97-033 (1997)

[2] CMS Collaboration, The Phase-2 Upgrade of the CMS Barrel Calorimeters Technical Design Report, CERN LHCC-2017-011 (2017). 